\section{Response to: Should we give long-term macrolide therapy for COPD?}

The letter submitted by Crosbie and Woodhead ${ }^{1}$ pertaining to our study of azithromycin for prevention of acute chronic obstructive pulmonary disease (COPD) exacerbations $^{2}$ contains a number of errors and misconceptions.

First, Crosbie and Woodhead stated that our study was not double-blind. The complete protocol (published as an on-line supplement to the manuscript) indicated that 'This is a prospective, randomised, double-blind, placebo-controlled trial.....

Second, while Crosbie and Woodhead correctly note that one-quarter of the patients we screened were excluded, and that the 'main reason for exclusion was cardiac issues', we suggest that their implication overstates the concern as only 102/1577 patients $(6 \%)$ were excluded for cardiac issues.

Third, Crosbie and Woodhead argue that the number of excluded patients '... could be further potentiated by the many other QT interval-prolonging drugs commonly taken by older patients'. One of the exclusion criteria listed in the manuscript was 'use of medications that prolong the QTc interval or are associated with torsades de pointes (with the exception of amiodarone)' and reference 33 is cited. This reference ${ }^{3}$ is the complete web-based list of medications known to prolong the QTc interval that was used to identify patients to exclude. Accordingly, there would be no 'further potentiation' of the effect as everyone excluded on the basis of taking any of these medications was already included in the 102 patients excluded for cardiac issues noted above.

Fourth, while Crosbie and Woodhead were correct in noting that macrolide resistance was higher in patients treated with azithromycin ( $81 \%$ vs $41 \%)$, they neglected to note that, because azithromycin markedly decreased the number of patients who were colonised with respiratory flora during the course of the study (66 vs 172), fewer patients receiving azithromycin actually became colonised with macrolide-resistant organisms than those receiving placebo (36 vs 49 ).

We encourage debate about whether the benefit of reducing acute COPD exacerbations with long-term azithromycin outweighs the potential associated risks, but suggest that the information used to frame this debate should be accurately presented.

\section{Richard K Albert}

Correspondence to Dr Richard K Albert, Department of Medicine, Denver Health, 777 Bannock MC 4000, Denver, CO 80204-4507, USA; ralbert@dhha.org

Funding This work was supported by National Heart Lung and Blood Institute grant number NHLBI 1 U10 HL074409-01. Supported conduct of study.

\section{Competing interests None.}

Provenance and peer review Not commissioned; internally peer reviewed.

To cite Albert RK, for the COPD Clinical Research Network. Thorax 2013;68:967.

Received 24 June 2013

Accepted 26 June 2013

Published Online First 13 July 2013

\section{SLinked}

http://dx.doi.org/10.1136/thoraxjnl-2013-203766

Thorax 2013;68:967.

doi:10.1136/thoraxjnl-2013-204089

\section{REFERENCES}

1 Crosbie PAJ, Woodhead M. Should we give long-term macrolide therapy for COPD? Thorax 2013;68:966.

2 Albert RK, Connett J, Bailey WC, et al. Azithromycin for prevention of exacerbations of COPD. $N$ Engl J Med 2011:365:689-98.

3 Arizona Center for Education and Resarch on Therapeutics. QT drug lists by risk groups. (http:// www.azcert.org/medical-pros/drug-lists/drug-lists. ffm). 